\title{
Lower Cardiovascular Risk with Diabetic Drugs: A Paradigm Shift from Glucocentricity to Cardio Protectiveness
}

\section{Bijaya Mohanty*}

Department of Medicine, Tata Main Hospital, Jamshedpur, India

\begin{abstract}
Cardiovascular disease is the leading cause of mortality and morbidity among diabetic patients. There by reducing cardiovascular risk is a major focus of diabetic management. International Diabetes Federation (IDF) in 2014 has projected that 592 million population will be affected by diabetes by the year 2035 from the present status of 387 million out of which $90 \%$ will have type 2 diabetes. The prevalence of diabetes and cardiovascular disease is growing exponentially. This rising tide of diabetes around the globe, the double jeopardy of diabetes and cardiovascular disease is infact a time bomb which may result in an explosion of these cardiovascular complications unless aggressive management of diabetes is done. While tight glycaemic control decrease the onset of microvascular complications evidence that it decreases macrovascular complications is limited. Lowering blood glucose levels in patients with diabetes mellitus is a too simplistic goal. The key component being how to lower blood sugar and how much. Diabetes drugs, even within the same "class" yield dramatically different cardiovascular outcomes. Infact a number of diabetic drugs may actually increase the risk of major cardiovascular events posing a challenge for the clinicians to select drugs for patients with type 2 diabetes mellitus. Thus, the treatment of type 2 diabetes needs to be invidualized and complex in which targeting cardiovascular risk factor is an important component. After the updated publication by Nissen and Wolski about the cardiovascular adverse outcomes of rosiglitazone both FDA (Food and Drug Administration) and European medicine agency (EMA) made it mandatory to have CVOT (Cardiovascular outcome trial) as an integral part of drug approval process. Therefore, the need of the hour is to evaluate anti diabetic drugs in relation to cardiovascular risk.
\end{abstract}

This review discusses the available evidence regarding cardiovascular safety of commonly used oral anti-diabetic medications.

Keywords: Diabetes mellitus; Diabetic drugs; Cardiovascular disease; Cardio-protectiveness

\section{Introduction}

Diabetes increases cardiovascular risk. Compared with subjects without diabetes people with diabetes have two to three-fold risk of heart disease [1]. $80 \%$ of death are attributable to cardiovascular causes. Infact diabetes is also considered as a coronary heart disease risk equivalent as the risk of cardiovascular mortality in type 2 diabetes mellitus is the same as in patients with previous myocardial infarction [2-5]. There by reducing cardiovascular risk is a major focus of diabetic management. International Diabetes Federation (IDF) in 2014 has projected that 592 million population will be affected by diabetes by the year 2035 from the present status of 387 million out of which $90 \%$ will have type 2 diabetes. The prevalence of diabetes and cardiovascular disease is growing exponentially. This rising tide of diabetes around the globe, the double jeopardy of diabetes and cardiovascular disease is infact a time bomb which may result in an explosion of these cardiovascular complications unless aggressive management of diabetes is done.

The therapeutic armamentarium to tackle type 2 diabetes is vast. We have biguanides, sulfonylureas, alpha-glucosidase inhibitors, Meglitinide analogues thiazolidinediones, dipeptidyl peptidase 4 inhibitors, glucagon-like peptide 1 analogues, SGLT2 Inhibitors (sodium glucose co transporter 2) and insulin in our basket for prescription. Till today our approach towards diabetes management is GLUCOCENTRIC. Does lowering of HBA1c (Glycosylated Haemoglobin) below a target of $6.5 \%$ to $7 \%$ translate in reduction of CVD (Cardiovascular disease) risk is a big question mark. The impact of conventional versus intensive glycaemic lowering strategies is still unclear. While tight glycaemic control decrease the onset of microvascular complications evidence that it decreases macrovascular complications is limited. Lowering blood glucose levels in patients with diabetes mellitus is a too simplistic goal.
The key component being how to lower blood sugar and how much. Diabetes drugs, even within the same "class" yield dramatically different cardiovascular outcomes. Several studies have suggested that some antidiabetic drugs increase CV risk, despite being effective at lowering blood glucose in type 2 diabetes [6-9]. There is a growing awareness that glycaemic efficacy of anti-diabetic drugs does not necessarily translate to cardiovascular safety. Over the past few years, there has been a number of trials evaluating the cardiovascular effects of antidiabetic drugs.

Therefore, the need of the hour is to evaluate anti diabetic drugs in relation to cardiovascular risk. To address this issue there is a change in FDA guideline 2008 and European Medicine Agency 2012 and it is mandatory now to have CVOT (cardiovascular outcome trial) as an integral part of drug approval process.

\section{Methods}

Literature search was performed using PubMed and Google Scholar using the keywords "cardiovascular risk" and "antidiabetic drugs." Additional references cited in these articles and regulatory guidelines were also included. We searched the Cochrane databases,

*Corresponding author: Bijaya Mohanty, Consultant, Department of Medicine, Tata Main Hospital, Jamshedpur, India, Tel: 91-7763807140; E-mail: bijayamohantytmh@gmail.com

Received: May 22, 2017; Accepted: June 04, 2017; Published: June 10, 2017

Citation: Mohanty B (2017) Lower Cardiovascular Risk with Diabetic Drugs: A Paradigm Shift from Glucocentricity to Cardio Protectiveness. Diabetes Case Rep 2: 123. doi: 10.4172/2572-5629.1000123

Copyright: @ 2017 Mohanty B. This is an open-access article distributed under the terms of the Creative Commons Attribution License, which permits unrestricted use, distribution, and reproduction in any medium, provided the original author and source are credited. 
major published trials, reviewed the existing data on the cardiovascular safety profiles of currently available oral diabetic drugs, compiled and presented here.

\section{Discussion}

\section{Biguanides}

Biguanides were introduced in late 1950s for treatment of type 2 diabetes. Metformin is the principal biguanide drug which is most commonly prescribed oral glucose-lowering agent worldwide and is recommended as first-line therapy by the American diabetes association (ADA), European association for the study of diabetes, and International Diabetes Federation [10]. Metformin has been used for over 50 years and it safety profile is well known [11]. The UK prospective diabetes study (UKPDS) a landmark study demonstrated a non-significant reduction $(p=0.052)$ in myocardial infarction/sudden death with intensive therapy. But there was a reduction in diabetes-related death and all-cause mortality in a sub-study of 342 overweight patients given metformin in whom there was $32 \%$ risk reduction in myocardial infarction/sudden death, $42 \%$ reduction in diabetes related death and $36 \%$ reduction in all-cause mortality rate. One meta-analysis in 2012 suggests that the use of metformin in younger patients and for longer periods of time may correlate better with $\mathrm{CV}$ event reduction as compared with placebo or no therapy [12]. In a retrospective observational study involving 5,631 patients with diabetes, the incidence of congestive heart failure (CHF) was lower over a 4.7-year follow-up period in patients using metformin compared to patients on sulfonylurea treatment. Metformin treatment did not increase the risk of developing CHF regardless of dose [13]. Patients with diabetes are more likely to have hypertension and higher lipid values and are more likely to be overweight than those without diabetes. Metformin may also have an effect on several CV risk factors, including lipid profile and blood pressure [14]. This drug has beneficial effect on lipid metabolism causing a decrease in total cholesterol LDL, triglycerides, APO B concentrations and by increasing HDL cholesterol level [15]. Metformin is also associated with weight loss and decrease in blood pressure. It also protects against vascular disease by virtue of its capacity to reduce inflammation and protection of endothelium [16].

\section{Sulfonylureas}

First generation drugs include acetohexamide, carbutamide, chlorpropamide, glycyclamide (tolhexamide), metahexamide, tolazamide and tolbutamide. Second generation drugs include glibenclamide (glyburide), glibornuride, gliclazide, glipizide, gliquidone, glisoxepide and glyclopyramide and the third-generation drugs which belongs to this category include glimepiride, although it is sometimes considered second-generation.

The diabetes mellitus, insulin glucose infusion in acute myocardial infarction (DIGAMI) study showed improved outcomes in patients treated with intravenous insulin-glucose infusions at the time of acute $\mathrm{MI}$ and demonstrated that the poorest outcomes were seen in patients who received sulfonylureas and no insulin-glucose infusions [17]. A Canadian retrospective population study showed an increase in the primary outcomes of all-cause mortality and fatal MI in patients treated with higher doses of first-generation sulfonylureas and glyburide but not metformin [18]. Sulfonylureas bind to sulfonylurea receptors (SUR) which are closely linked to ATP dependent K channels. Some bind to SUR1 receptors in beta cells of pancreas to exert insulinotropic effects and some bind to SUR2A/B receptors located in myocardium and coronary smooth muscles there by preventing development of protective ischaemic preconditioning [19]. The first-generation sulfonylureas, including tolbutamide, possess lower pancreatic affinity and thus are more likely to bind cardiac receptors and interfere with cardiac ischemic preconditioning [20,21]. The second-generation sulfonylureas, glimepiride, glyburide available as glibenclamide, glipizide, and gliclazide have a lower affinity for $\mathrm{CV}$ tissue and may have fewer unfavorable effects, although not all data have been consistent. Glibenclamide is shown to be harmful to patients with type 2 diabetes mellitus and CAD, even when combined with metformin, and avoiding the drug is suggested in such high-risk patients [22]. CV mortality, nonfatal MI, and risk of mortality are increased in monotherapy with glimepiride, glibenclamide, gliclazide, and tolbutamide compared with metformin, suggesting that sulfonylureas may not be the best option for the initial management of patients with diabetes who are at risk for $\mathrm{CV}$ events [23]. The newer sulfonylurea gliclazide has been suggested as a better sulfonylurea agent to use for the treatment of type 2 diabetes mellitus. While previous evidence from the Action in Diabetes and Vascular Disease: Preterax and Diamicron Modified Release Controlled Evaluation (ADVANCE) trial reveals that intensive glucose control with gliclazide has no significant effect on major macrovascular events, more recent evidence shows gliclazide to be the only sulfonylurea associated with a lower risk of CV events and mortality-similar to metformin [24-26].

\section{Thiazolidinediones}

The thiazolidinediones also known as glitazones, are a class of medications used in the treatment of diabetes mellitus type 2 . They were introduced in the late 1990s. They contain a functional group in which thiazolidine serves as a dione. They act by activating PPARs (peroxisome proliferator-activated receptors), with greatest specificity for PPAR $\gamma$ (PPAR-gamma). Troglitazone was the 1st agent but was withdrawn from market in 1999 for hepatotoxicity. At the same time Rosiglitazone was approved in 1999 and became one of the 25 best selling drugs that year. However, in 2007 Nissen and Wolski published the results of their meta-analysis of the long term cardiovascular risk of rosiglitazone from 42 randomized clinical trials [27]. As compared with the control group the odds ratio for myocardial infarction was 1.43 (95\%CI 1.08-1.98; P-0.03) and 1.64(95\%CI, 0.892.74; P-0.06) for death from cardiovascular causes with rosiglitazone. This raised questions regarding overall therapeutic benefit of rosiglitazone. After the updated publication by Nissen and Wolski rosiglitazone was withdrawn in 2010 from Europe on recommendation of EMEA but continued to have restricted availability in US and other countries. Then came Pioglitazone and was found to be comparatively cardio protective. But it increases risk of heart failure although there is no increase in associated mortality [28]. This is believed to be a class effect of thiazolidinediones and these drugs should be avoided in patients with congestive heart failure [29]. The prospective pioglitazone clinical trial in macrovascular events (PROactive) study shown that in type 2 diabetes mellitus patients at high risk for macrovascular events pioglitazone significantly reduced secondary end points composed of death and non-fatal myocardial infarction [30].

\section{Alpha-glucosidase inhibitors}

Alpha glucosidase inhibitors are used to establish greater glycaemic control over hyperglycaemia in type 2 diabetes mellitus, particularly with regard to postprandial hyperglycaemia. They may be used as monotherapy in conjunction with an appropriate diabetic diet and exercise, or they may be used in conjunction with other anti-diabetic drugs. The alpha glucosidase inhibitors (AGIs) lower blood glucose through competitive blockade of intestinal alpha glucosidases, which convert complex carbohydrates into monosaccharides. This results in a modified intestinal absorption of carbohydrates and consequently a slower rise in post-prandial blood glucose [31]. Available agents 


\begin{tabular}{|c|c|c|c|c|}
\hline Trials & EXAMINE & SAVOR TIMI & TELCOS & CARMELINA \\
\hline No of patients enrolled & 5380 & 16492 & 14724 & 8300 \\
\hline Drug used & Allogliptin & Saxagliptin & Sitagliptin & Linagliptin \\
\hline Year of completion & 2013 & 2013 & 2014 & 2018 \\
\hline Comparator & Placebo & Placebo & Placebo & Placebo \\
\hline Result & $\begin{array}{l}\text { Non-Inferior for primary } \\
\text { composite end points } \\
\text { CVD risk-same as placebo }\end{array}$ & $\begin{array}{l}\text { Non-Inferior neither reduced nor increased the risk } \\
\text { of the primary composite endpoint of } \mathrm{CV} \text { death, MI, } \\
\text { or ischemic stroke }\end{array}$ & $\begin{array}{l}\text { No CVD Risks or heart failure with } \\
\text { sitagliptin in high-risk diabetic patients }\end{array}$ & Ongoing \\
\hline
\end{tabular}

Table 1: EXAMINE-Examination of cardiovascular outcomes with Alogliptin versus standard of care; SAVOR-Saxagliptin assessment of vascular outcomes recorded in patients with diabetes mellitus; TECOS-To evaluate cardiovascular outcomes after treatment with sitagliptin; CARMELINA-Cardiovascular and renal microvascular outcome study with Linagliptin in patients with type 2 diabetes mellitus.

include acarbose, miglitol and voglibose. A Cochrane meta-analysis reported a $0.8 \% \mathrm{HbA} 1 \mathrm{c}$ reduction and no clinically relevant effects on lipids or body weight when acarbose, the most widely prescribed AGI, was compared to placebo [32]. In addition, acarbose may also have beneficial effects on endothelial function by obtunding postprandial glucotoxicity [33]. Miglitol has also been shown to reduce waist circumference, and in particular visceral fat, in patients with metabolic syndrome [34]. There are no long-term studies examining the effect of AGIs on cardiovascular disease or mortality in T2DM. Although not initially powered to draw conclusions on cardiovascular outcomes, acarbose treatment was also associated with a reduction in the development of the composite outcome of cardiovascular events, which includes cardiovascular death, MI, stroke, heart failure, peripheral vascular disease and revascularization (HR 0.51, 95\% CI $0.28-0.95, \mathrm{p}=0.03)$ [35].

\section{Incretin-Based Drugs}

\section{Dipeptidyl peptidase-4 inhibitors}

DPP-4 inhibitors are a class of oral hypoglycaemics that block DPP4. The first agent of the class sitagliptin was approved by the FDA in 2006. Then came saxagliptin, linagliptin alogliptin. Vildagliptin, teneligliptin etc. The DPP-4 inhibitors, otherwise known as the gliptins, are generally considered to have a neutral effect on weight [36]. It has been suggested that the modulation of endothelial progenitor cells, inflammatory pathways and ischemic response are the major cardiovascular targets of gliptins [37]. The Saxagliptin assessment of vascular outcomes recorded in patients with diabetes mellitus thrombolysis in myocardial infarction(SAVOR-TIMI) study evaluated 16,492 patients of T2DM who had a history of or were at risk for cardiovascular events that received either saxagliptin or placebo which showed no alteration in ischaemic events but it increased the rate of hospitalization for heart failure patients [38]. The examination of CV outcomes with allogliptin versus standard of care(EXAMINE) study, a randomized double- blind trial with 5380 patients of T2DM who required hospitalization during the previous 15-90 days due to either acute MI or unstable angina. Allogliptin did not increase MACE including MI or heart failure compared to patients receiving placebo [39]. The TELCOS trial also evaluated the effectiveness of sitagliptin and found that this therapy did not increase adverse cardiovascular outcomes [40]. The cardiovascular safety and renal microvascular outcome study (CARMELINA) with linagliptin in patients with type2 diabetes will last till 2018, will assess the long-term impact on cardiovascular mortality, morbidity and renal function with linagliptin [41]. The DPP-4 inhibitors are safe in terms of hard cardiovascular endpoints, but their effect on the risk of heart failure remains uncertain. The clinical significance of the finding of an early increased hospitalization for heart failure with saxagliptin in the SAVOR study is unclear, although it is unlikely to be a class effect. The FDA safety review recommends considering discontinuation of specifically saxagliptin or alogliptin in patients who develops heart failure [42]. Overall the results of the trials with gliptins are encouraging (Table 1).

\section{Glucagon-like peptide-1 agonists}

Currently available agents include: exenatide, liraglutide, albiglutide, lixisenatide, and dulaglutide. All are FDA-approved with the exception of lixisenatide, which is approved in Europe. GLP-1 agonists, when compared to placebo, reduced $\mathrm{HbAlc}$ by about $1 \%$ and resulted in $1.5 \mathrm{~kg}$ to $2.5 \mathrm{~kg}$ weight loss over 30 weeks [43]. In addition, treatment with GLP-1 agonists have been shown to further favorably alter the metabolic profile through modest reductions in low-densitylipoprotein cholesterol, total cholesterol and triglycerides [44], as well as reductions in systolic blood pressure (weighted mean difference -2.22 mmHg; $95 \%$ CI -2.97 to -1.47 ), although this may be accompanied by a compensatory increase in heart rate [45]. The cardioprotective effects of GLP-1 agonists have been well documented in pre-clinical studies. In the clinical setting, similar positive effects have been observed to various degrees in pilot studies, and the mechanism was thought to be related to reduced apoptosis and nuclear oxidative stress and improvement in myocardial glucose metabolism [46].

The evaluation of lixisenatide in acute coronary syndrome (ELIXA) trial randomized 6068 patients with T2DM and an acute coronary event within the last 180 days to receive lixisenatide or placebo on top of standard of care. After a median follow-up of 25 months, there was no difference in the primary composite endpoint of cardiovascular death, non-fatal MI, non-fatal stroke, or hospitalization for unstable angina between groups (13.4 vs $13.2 \%$, HR 1.02 , 95\% CI $0.89-1.17$, $\mathrm{p}=0.81$ for superiority, $\mathrm{p}<0.001$ for non-inferiority), and no difference in heart failure hospitalizations ( 4.0 vs $4.2 \%$, HR 0.96 , 95\% CI 0.75 1.23) [47]. $\mathrm{n}$ the Liraglutide effect and action in diabetes: evaluation of cardiovascular outcome results (LEADER) trial, which randomized 9340 T2DM patients with high cardiovascular risk, liraglutide reduced the primary composite endpoint of cardiovascular death, non-fatal MI or non-fatal stroke compared to placebo after a median follow-up of 3.8 years ( 13 vs $14.9 \%$, HR $0.8,95 \%$ CI $0.78-0.97, \mathrm{p}<0.001$ for noninferiority; $\mathrm{p}=0.01$ for superiority) [48].

\section{Meglitinides}

The meglitinides have similar action to sulfonylureas but are pharmacologically distinct and bind to different receptors on pancreatic KATP channels. Currently available meglitinides include repaglinide and nateglinide. There are currently no long-term studies of meglitinides to assess cardiovascular outcomes or mortality in T2DM, although the Nateglinide and Valsartan in Impaired Glucose Tolerance Outcomes Research (NAVIGATOR) study showed no difference between nateglinide and placebo with respect to the core composite cardiovascular outcome [49]. 


\begin{tabular}{|c|c|c|c|c|}
\hline Study & Drug & Comparator & Primary endpoint \\
\hline CANVAS & Canagliflozin & Placebo & Major adverse cardiovascular events, including CV death, non-fatal MI, and non-fatal stroke & 2017 \\
\hline CARMELINA & Linagliptin & Placebo & $\begin{array}{c}\text { Composite endpoint: Cardiovascular death, non-fatal myocardial infarction, non-fatal stroke } \\
\text { and hospitalization for unstable angina pectoris }\end{array}$ \\
\hline EXSCEL & Exenatide once weekly & Placebo & Composite endpoint: Cardiovascular death, non-fatal MI, or non-fatal stroke \\
\hline REWIND & Dulaglutide & Placebo & Composite endpoint: Cardiovascular death, non-fatal MI, or non-fatal stroke \\
\hline DECLARE-TIMI 58 & Dapagliflozin & Placebo & Composite endpoint: CV death, MI or ischemic stroke \\
\hline CAROLINA & Linagliptin & Glimepiride & Composite endpoint: CV death, non-fatal MI (excluding silent MI), non-fatal stroke and \\
hospitalisation for unstable angina
\end{tabular}

Table 2: Upcoming cardiovascular outcomes trials in type 2 diabetes.

\section{Sodium glucose cotransporter-2 inhibitors}

Another new group of drugs the SGLT2 (sodium glucose co transporter 2) inhibitors hit the market in 2013 onwards. FDA and EDA have approved three inhibitors, Canagliflozin in 2013, Empagliflozin 2014 and Dapagliflozin in 2014 with several other under late stage clinical development. Ipragliflozin, tofoglifozin and luseogliflozinhave been approved in Japan. They act by reducing re-absorption of glucose in the kidney and increasing urinary glucose excretion there by lowering plasma glucose levels along with weight loss. It blocks approximately $50 \mathrm{~g}$ to $80 \mathrm{~g}$ of glucose per day from being reabsorbed. The EMPAREG trial using empagliflozin in 592 sites in 42 countries shown that empagliflozin modulates cardiovascular risk factors. It decreases blood pressure and arterial stiffness, decreases weight specially the visceral adiposity. It decreases sympathetic nervous system activity and oxidative stress. It also decreases albuminuria and uric acid level. It modifies lipid profile by decreasing LDL and triglycerides along with increasing HDL level. This class of drugs has a low potential to induce hypoglycaemia unless used with sulfonylureas or insulin. Empagliflozin displayed a $38 \%$ reduction in cardiovascular death and a $32 \%$ reduction in all-cause mortality. The multicentre trial to evaluate the effect of dapagliflozin on the incidence of cardiovascular events (DECLARE TIMI 58) is expected to be finished by 2019 , designed to evaluate the effect of dapagliflozin on the incidence of cardiovascular events [50]. The CANVAS trial is also designed to study the effect of cardiovascular safety of canagliflozin in patients with type 2 diabetes. This study is expected to complete data collection by 2017 . Recent reports have suggested that use of SGLT2 inhibitors may increase the risk of diabetic ketoacidosis [51]. The pathophysiological mechanisms of increased risk of diabetic ketoacidosis are not well defined till now. So, selection of patients for this drug should be made carefully. However, the cardiovascular protection of these drugs may have a huge impact on the clinical practice guidelines in future and It may be considered as the 1st line drug in the treatment of type 2 diabetes.

\section{CVOT (Cardiovascular outcome trials)}

Although cardiovascular disease is the cause of death in $75 \%$ of diabetics, there exist no well-designed, adequately-powered comparative effectiveness trials evaluating macrovascular outcomes for diabetes drugs. The absence of information on macrovascular effects of diabetes therapies is the unfortunate consequence of current regulatory policy that emphasizes the importance of glucose lowering, not health outcomes, as a therapeutic goal. Pre-approval studies focus on demonstrating maximal glucose lowering effects. Patients are selected with relatively high HbAlclevels to enhance apparent "efficacy." Even the pharmaceutical industry came with "bragging" rights- "my drug lowers blood sugar more than your drug." Patients at high CV risk are deliberately avoided. Therefore, Clinical outcomes trials are essential to determine optimal approach to prevent CV morbidity and mortality. To address this issue there is a change in FDA guideline 2008 and European Medicine Agency 2012 and it is mandatory now to have CVOT (cardiovascular outcome trial) as an integral part of drug approval process [52-54]. The upcoming cardiovascular trials in type 2 diabetes mellitus are listed below (Table 2).

\section{Conclusion}

As can be seen from the various trials reviewed here, favourable glycaemic efficacy does not necessarily translate to favourable cardiovascular outcomes. Cardiovascular safety needs to be prioritized over glucocentricity. This paradigm shift from glucocentricity to cardio-protectiveness poses a challenge to the practitioners to balance between the benefit of glycaemic control and the inherent risk in glucose lowering medications.

As the diabetic patients are at high risk for cardiovascular morbidity and mortality the knowledge of cardioprotective effects of diabetic drugs is important for the clinicians dealing with this condition and translating it in patient care.

\section{Recommendation}

Based on current evidence, metformin should remain the firstline drug of choice in T2DM, being the most extensively studied and demonstrating excellent cardiovascular safety even with long term use. Although evidence for the cardiovascular safety of sulfonylureas are inconsistent, the first-generation agents are probably associated with net harm and should be avoided. Newer generation sulfonylureas have a comparatively better cardiovascular profile, but weight gain remains a problem. The meglitinides and AGIs lack cardiovascular safety data in T2DM and should therefore be reserved as second-line agents. Among the TZDs pioglitazone may have beneficial cardiovascular effects but contraindicated in heart failure. The incretin-based drugs have been at the forefront in the era of cardiovascular safety trials and have been extensively studied. Current evidence suggests that the gliptins have neutral cardiovascular effect, but may increase risk of heart failure, particularly saxagliptin. Among the GLP-1 agonists, liraglutide may have beneficial effects on cardiovascular outcomes, but this requires further validation. Similarly, the SGLT-2 inhibitors have shown promising results with empagliflozin and may potentially confer cardiovascular benefits, although additional data is needed to substantiate this. Several large ongoing randomized trials whose results are expected in the coming years. This will definitely guide the clinicians to optimize the treatment for their diabetic patients.

\section{References}

1. Gotto AM (2007) Cardiologists role in improving glucose control and global cardiovascular risks in patients with type 2 diabetes mellitus. Am J Cardiol 99 3B-5B.

2. Haffner SM, Lehto S, Ronnemaa T, Pyorala K, Laakso M (1998) Mortality from coronary heart disease in subjects with type 2 diabetes and non-diabetic subjects and without prior myocardial infarction. N Engl J Med 339: 229-234.

3. Juutilainen A, Lehto S, Ronnemaa T, Pyorala K, Laakso M (2008) Type 2 diabetes is a coronary heart disease equivalent: An 18-year prospective population based study in Finnish subjects. Diabetes care 28: 2901-2907. 
4. Goldberg RB (2003) Cardiovascular disease in patients who have diabetes Cardiol Clin 21: 399-413.

5. Grundy SM, Benjamin IJ, Burke GL (1999) Diabetes and cardiovascular disease: A statement for healthcare professionals from the American Heart Association. Circulation 100: 1134-1146.

6. Graham DJ, Ouellet-Hellstrom R, MaCurdy TE, Ali F, Sholley C, et al. (2010) Risk of acute myocardial infarction, stroke, heart failure, and death in elderly Medicare patients treated with rosiglitazone or pioglitazone. JAMA 304: 411-418.

7. Nissen SE, Wolski K (2007) Effect of rosiglitazone on the risk of myocardial infarction and death from cardiovascular causes. N Engl J Med 356: 2457-2471.

8. Lago RM, Singh PP, Nesto RW (2007) Congestive heart failure and cardiovascular death in patients with prediabetes and type 2 diabetes given thiazolidinediones: A meta-analysis of randomised clinical trials. Lancet 370 : 1129-1136.

9. Goldfine $A B$ (2008) Assessing the cardiovascular safety of diabetes therapies. N Engl J Med 359: 1092-1095.

10. Inzucchi SE, Bergenstal RM, Buse JB, Diamant M, Ferrannini E, et al. (2015) Management of hyperglycemia in type 2 diabetes, 2015: A patient-centered approach. Update to a position statement of the American diabetes association (ADA) and the European association for the study of diabetes (EASD). Diabetes Care 38: 140-149.

11. DeFronzo RA, Goodman AM (1995) Efficacy of metformin in patients with noninsulin-dependent diabetes mellitus. The multi-center metformin study group. $\mathrm{N}$ Engl J Med 333: 541-549.

12. Lamanna C, Monami M, Marchionni N, Mannucci E (2011) Effect of metformin on cardiovascular events and mortality: A meta-analysis of randomized clinical trials. Diabetes Obes Metab 13: 221-228.

13. McAlister FA, Eurich DT, Majumdar SR, Johnson JA (2008) The risk of heart failure in patients with type 2 diabetes treated with oral agent monotherapy. Eur $\mathrm{J}$ Heart Fail 10: 703-708.

14. Singh S, Bhat J, Wang PH (2013) Cardiovascular effects of anti-diabetic medications in type 2 diabetes mellitus. Curr Cardiol Rep 15: 327-335.

15. Charles MA, Eschwege E, Grandmottet P (2000) Treatment with metformin of non-diabetic men with hypertension, hyper triglyceridaemia and central fat distribution: the BIGPRO 1.2 trial Diabetes. Metab Res Rev 16: 2-7.

16. De Jager J, Kooy A, Schalkwijkc (2014) Long term effects of metformin on endothelial function in type 2 diabetes: A randomized controlled trial. J Intern Med 275: 59-70.

17. Malmberg K (1997) Prospective randomised study of intensive insulin treatment on long term survival after acute myocardial infarction in patients with diabetes mellitus. DIGAMI (Diabetes Mellitus, insulin glucose infusion in acute myocardial infarction) study group. BMJ 314: 1512-1515.

18. Simpson SH, Majumdar SR, Tsuyuki RT, Eurich DT, Johnson JA (2006) Doseresponse relation between sulfonylurea drugs and mortality in type 2 diabetes mellitus: A population-based cohort study. CMAJ 174: 169-174.

19. Meier JJ, Gallwicz B, Schmidt WE, Mugge A, Nauck MA (2004) Is impairment of ischaemic preconditioning by sulfonylurea drugs clinically important? Heart 90: 9-12

20. Panicker GK, Karnad DR, Salvi V, Kothari S (2012) Cardiovascular risk of oral antidiabetic drugs: current evidence and regulatory requirements for new drugs. J Assoc Physicians India 60: 56-61.

21. Juurlink DN, Gomes T, Shah BR, Mamdani MM (2012) Adverse cardiovascular events during treatment with glyburide (glibenclamide) or gliclazide in a highrisk population. Diabet Med 29: 1524-1528.

22. Fisman EZ, Tenenbaum A (2009) A cardiologic approach to non-insulin antidiabetic pharmacotherapy in patients with heart disease. Cardiovasc Diabetol 20: 8-38.

23. Schramm TK, Gislason GH, Vaag A (2011) Mortality and cardiovascular risk associated with different insulin secretagogues compared with metformin in type 2 diabetes, with or without a previous myocardial infarction: a nationwide study. Eur Heart J 32: 1900-1908.

24. Patel A, MacMahon S, Chalmers J (2008) Intensive blood glucose contro and vascular outcomes in patients with type 2 diabetes. N Engl J Med 358: 2560-2572.
25. Zeller M, Danchin N, Simon D (2010) Impact of type of preadmission sulfonylureas on mortality and cardiovascular outcomes in patients with diabetes with acute myocardial infarction. J Clin Endocrinol Metab 95: 4993-5002.

26. Schramm TK, Gislason GH, Vaag A (2011) Mortality and cardiovascular risk associated with different insulin secretagogues compared with metformin in type 2 diabetes, with or without a previous myocardial infarction: A nationwide study. Eur Heart J 32: 1900-1908.

27. Schramm TK, Gislason GH, Vaag A, Rasmussen JN, Folke F, et al. (2012) Erratum in mortality and cardiovascular risk associated with different insulin secretagogues compared with metformin in type 2 diabetes, with or without a previous myocardial infarction: A nationwide study. Eur Heart J 33: 1183.

28. Nissen SE, Wolski K (2007) Effect of rosiglitazone on the risk of myocardia infarction and death from cardiovascular causes. N Engl J Med 356: 2457-2471.

29. Lincoff AM, Wolski K, Nicholls SJ, Nissen SE (2007) Pioglitazone and risk of cardiovascular events: A meta-analysis of randomized trials. J Am Med Assoc 298: 1180-1198.

30. FDA guidance for industry. Diabetes mellitus-Evaluation of cardiovascular risk in new antidiabetic therapies to treat type 2 diabetes; 2008

31. Dormandy JA, Charbonnel B, Eckland DJ, Erdmann E, Massi-Benedetti M, et al. (2005) Secondary prevention of macrovascular events in patients with type 2 diabetes in the PROactive study: A randomized controlled trial Lancet 366 : 1279-1289.

32. DiNicolantonio JJ, Bhutani J, O'Keefe JH (2015) Acarbose: Safe and effective for lowering postprandial hyperglycaemia and improving cardiovascular outcomes. Open Heart 2: e000327.

33. Van de Laar FA, Lucassen PL, Akkermans RP, Van de Lisdonk EH, Rutten GE et al. (2005) Alpha-glucosidase inhibitors for type 2 diabetes mellitus. Cochrane Database Syst Rev 2: CD003639.

34. DiNicolantonio JJ, Bhutani J, O'Keefe JH (2015) A carbose: Safe and effective for lowering postprandial hyperglycaemia and improving cardiovascular outcomes. Open Heart 2: e000327.

35. Shimabukuro M, Higa M, Yamakawa K, Masuzaki H, Sata M (2013) Miglitol, alpha-glycosidase inhibitor, reduces visceral fat accumulation and cardiovascular risk factors in subjects with the metabolic syndrome: A randomized comparable study. Int J Cardiol 167: 2108-2113.

36. Chiasson JL, Josse RG, Gomis R (2003) A carbose treatment and the risk of cardiovascular disease and hypertension in patients with impaired glucose tolerance: The STOP-NIDDM trial. JAMA 290: 486-494.

37. Davidson JA (2009) Advances in therapy for type 2 diabetes: GLP-1 recepto agonists and DPP-4 inhibitors. Cleve Clin J Med 76: S28-38.

38. Fadini GP, Avogaro A (2011) Cardiovascular effects of DPP-4 inhibition: Beyond GLP-1. Vasc Pharmacol 55: 10-16.

39. Scirika BM, Bhatt DL, Braunwald E (2013) Saxagliptin and cardiovascular outcomes in patients with type 2 diabetes mellitus. N Engl J Med 369: 1317-1326.

40. White WB, Cannon CP, Heller SR, Nissen SE, Bergenstal RM, et al. (2013) Allogliptin after acute coronary syndrome in patients with type 2 diabetes. $\mathrm{N}$ Engl J Med. 369: 1327-1335.

41. Green GB, Bethel MA, Armstrong PW (2015) Effect of Sitagliptin on cardiovascular outcomes in type 2 diabetes N Engl J Med 373: 232-242.

42. Clinical trials Gov (2013) cardiovascular and renal microvascular outcome study with linagliptin in patients with type 2 diabetes mellitus (CARMELINA); 2017.

43. FDA drug safety communication: FDA adds warnings about heart failure risk to labels of type 2 diabetes medicines containing saxagliptin and alogliptin; 2016

44. Hyangdan DS, Royle P, Clar C, Sharma P, Waugh N, et al. (2011) Glucagon-like peptide analogues for type 2 diabetes mellitus. Cochrane Database Syst Rev 10: CD006423

45. Sun F, Wu S, Wang J, Guo S, Chai S, et al. (2015) Effect of glucagon-like peptide-1 receptor agonists on lipid profiles among type 2 diabetes: $A$ systematic review and network meta-analysis. Clin Ther 37: 225-241.

46. Katout M, Zhu H, Rutsky J, Shah P, Brook RD, et al. (2014) Effect of GLP 1 mimetics on blood pressure and relationship to weight loss and glycemia lowering: Results of a systematic meta-analysis and meta-regression. Am J Hypertens. 27: 130-139. 
Citation: Mohanty B (2017) Lower Cardiovascular Risk with Diabetic Drugs: A Paradigm Shift from Glucocentricity to Cardio Protectiveness. Diabetes Case Rep 2: 123. doi: 10.4172/2572-5629.1000123

Page 6 of 6

47. Angeli FS, Shannon RP (2014) Incretin-based therapies: can we achieve glycaemic control and cardioprotection? J Endocrinol 221: T17-30.

48. Pfeffer MA, Claggett B, Diaz R, Dickstein K, Hertzel C, et al. (2015) Lixisenatide in patients with type 2 diabetes and acute coronary syndrome. N Engl J Med. 373: $2247-2257$

49. Marso SP, Daniels GH, Brown-Frandsen K, Kristensen P, Mann JF, et al. (2016) Liraglutide and cardiovascular outcomes in type 2 diabetes. N Engl J Med. 375 : 311-322.

50. Holman RR (2010) Effect of Nateglinide on the incidence of diabetes and cardiovascular events. N Engl J Med 362: 1463-1476.

51. Clinical trials Gov (2015) Multicentre trial to evaluate the effects of Dapagliflozin on the incidence of cardiovascular events (Declare TIMI58).

52. Brooks M (2015) SGLT2 inhibitors diabetes drugs may cause ketoacidosis: FDA.

53. Guidance for Industry: Diabetes mellitus-Evaluating cardiovascular risk in new antidiabetic therapies to treat type 2 diabetes; 2008.

54. Guideline on clinical investigation of medicinal products in the treatment of diabetes mellitus; 2010. 